\title{
Preparação de um material de referência certificado para controle de agrotóxicos em hortifrutigranjeiros: estudo da homogeneidade
}

\author{
Preparation of a certified reference material for pesticide control \\ in the cultivation of fruits and vegetables: a homogeneity study \\ Maria Helena Wohlers Morelli CARDOSO ${ }^{1 *}$, Armi Wanderley da NÓBREGA ${ }^{1}$, \\ Hélio de Carvalho VITAL ${ }^{2}$, Shirley ABRANTES ${ }^{1}$
}

\section{Resumo}

Neste trabalho são apresentados os esforços para garantir a homogeneidade de um material de referência. Níveis residuais (mg.kg-1) de quatro agrotóxicos $(\gamma-\mathrm{HCH}$, fenitrotiona, clorpirifós e procimidona) foram adicionados à polpa de tomate com o objetivo de se preparar um material de referência certificado. As propriedades mais importantes desses materiais são a homogeneidade e a estabilidade. Antes de serem enviados a outros laboratórios, os materiais de referência precisam ter sua homogeneidade verificada. Nas etapas prévias, amostras foram avaliadas de modo a prover dados sobre o tratamento mais adequado para minimizar a variabilidade analítica do lote preparado e garantir a qualidade da amostra candidata a material de referência certificado, tão bem como a estimativa da incerteza associada à homogeneidade. A análise de variância fornece informações sobre a variabilidade do lote preparado e o grau de invariabilidade da amostra fortificada. Depois da preparação, as amostras foram expostas à radiação gama na dose de $2 \mathrm{kGy}$ e submetidas a um estudo interlaboratorial para certificação. As concentrações certificadas dos agrotóxicos após caracterização foram 0,191 $\pm 0,047 \mathrm{mg} \cdot \mathrm{kg}^{-1} ; 0,192 \pm 0,068 \mathrm{mg} \cdot \mathrm{kg}^{-1} ; 0,225 \pm 0,076 \mathrm{mg} \cdot \mathrm{kg}^{-1} \mathrm{e}$ $0,177 \pm 0,051 \mathrm{mg} \cdot \mathrm{kg}^{-1}$ para o $\gamma-\mathrm{HCH}$, a fenitrotiona, clorpirifós e procimidona respectivamente.

Palavras-chave: agrotóxicos; tomate; material de referência; homogeneidade.

\begin{abstract}
This study reports on the efforts to ensure homogeneity of certified reference materials. Residues of four pesticides $(\gamma$-HCH, fenitrothion, chlorpyrifos, and procymidone) were added to tomato pulp in order to produce reference materials. The two most important properties of such materials are known to be homogeneity and stability. Before being shipped to other laboratories, reference materials must have their homogeneity certified. As part of a preliminary study, hundreds of samples were investigated in order to provide data on the most suitable treatments to minimize analytical variability and ensure the quality of samples intended for use as reference materials as well as to yield estimates of the uncertainty associated to the homogeneity data. In addition, the variance analysis provided accurate information on the bulk variability and the degree of non-homogeneity of contaminated samples. After preparation, the samples were exposed to $2 \mathrm{kGy}$ of gamma radiation and submitted to an interlaboratory certification study. The certified concentrations of $\gamma$-HCH, fenithrotion, chlorpyrifos, and procymidone were $0.191 \pm 0.047 \mathrm{mg} \cdot \mathrm{kg}^{-1} ; 0.192 \pm 0.068 \mathrm{mg} \cdot \mathrm{kg}^{-1} ; 0.225 \pm 0.076 \mathrm{mg} \cdot \mathrm{kg}^{-1}$, and $0.177 \pm 0.051 \mathrm{mg} . \mathrm{kg}^{-1}$, respectively.

Keywords: pesticides; tomato; reference material; homogeneity.
\end{abstract}

\section{Introdução}

Materiais de referência (MR) e materiais de referência certificados (MRC) são as bases para verificação da exatidão de medições analíticas, visando a garantir a sua confiabilidade.

Infelizmente, não existem MR e MRC para todas as análises químicas realizadas em laboratórios, sendo que os mesmos se encontram disponíveis apenas para as técnicas analíticas rotineiramente empregadas e para um número muito reduzido de matrizes. Tais materiais são muito caros, tendo em vista que as etapas de certificação são demoradas e dispendiosas. Poucos são produzidos no Brasil e, em sua maioria, são produzidos por países como os EUA, Inglaterra, Alemanha, Austrália entre outros (ALVES; MORAES, 2002).
$\mathrm{Na}$ análise de agrotóxicos existe, ainda, um grande inconveniente analítico que é o efeito da matriz e, considerandose que alguns métodos sofrem interferência da mesma, tornase necessário utilizar um material semelhante à matriz da amostra (ZUCCHINI, 2002). No Brasil, não existe nenhum provedor de MRC para agrotóxicos, o que dificulta o acesso e, conseqüentemente, aumenta o custo para o laboratório. São disponíveis apenas MR destinados a Ensaios de Proficiência (BASTOS et al., 2007).

A produção de um MRC requer um planejamento experimental detalhado, no qual deve ser prevista uma quantidade suficiente de material para a execução de todos os

Recebido para publicação em 26/5/2008

Aceito para publicação em 20/6/2009 (003550)

Departamento de Química, Instituto Nacional de Controle de Qualidade em Saúde, Fundação Oswaldo Cruz - INCQS/FIOCRUZ, Av. Brasil 4365, CEP 21045-900, Manguinhos,

Rio de Janeiro - RJ, Brasil, E-mail: helenawohlers@hotmail.com

${ }^{2}$ Divisão de Defesa Química, Biológica e Nuclear, Centro Tecnológico do Exército - DDQBN/CTEx, Av. da Américas, CP 28705, CEP 23020-470, Guaratiba, Rio de Janeiro - RJ, Brasil

${ }^{*}$ A quem a correspondência deve ser enviada 
estudos inerentes a ele (INTERNATIONAL ORGANIZATION FOR STANDARDIZATION, 2006). Basicamente, são quatro etapas de trabalho para a produção de um MRC: preparo do material; envasamento das amostras, com verificação da homogeneidade do material nos frascos; estabelecimento da estabilidade, que garantirá ao material embalado ser mantido íntegro durante estocagem por tempo pré-estabelecido e a certificação dos valores atribuídos às propriedades de interesse do material preparado. A embalagem desse material também tem relevância, pois irá contribuir para manter sua integridade durante todo o período despendido no transporte e armazenamento. $\mathrm{O}$ valor de propriedade certificada dos materiais de referência deve ser mensurado a partir de cálculos estatísticos adequados e informado com suas incertezas devidamente estimadas, provenientes da caracterização da amostra, da homogeneidade e da estabilidade da mesma (INTERNATIONAL ORGANIZATION FOR STANDARDIZATION, 2006; INTERNATIONAL LABORATORY ACCREDITATION COOPERATION, 2000; CHUI; IAMASHITA; BISPO, 2005).

O teste de homogeneidade da amostra é um dos fatores essenciais para a garantia da manutenção das propriedades físico-químicas do material estudado e, portanto, o número de recipientes, retirados do lote preparado, selecionados para esta avaliação deve ser representativo em relação ao quantitativo final. Para o teste, o número de frascos selecionados aleatoriamente deve incluir entre 10 e 30 unidades do lote preparado (INTERNATIONAL ORGANIZATION FOR STANDARDIZATION, 2006). O planejamento para verificar a homogeneidade de um lote de material preparado para fins de certificação deve indicar as variabilidades da amostra dentro do recipiente, assim como entre os recipientes que contêm os materiais que compõem o lote, devidamente envasados (INTERNATIONAL ORGANIZATION FOR STANDARDIZATION, 2006; INTERNATIONAL LABORATORY ACCREDITATION COOPERATION, 2000). Os dados para o teste de homogeneidade devem ser executados em condições de estrita repetitividade (mesmo laboratório, mesmo analista e todas as amostras testadas no mesmo dia, se possível). Além disso, na variabilidade entre os frascos, deve ser incluída a incerteza relativa a tal condição (INTERNATIONAL ORGANIZATION FOR STANDARDIZATION, 2006; INTERNATIONAL LABORATORY ACCREDITATION COOPERATION, 2000; ELLISON et al, 2001).

Assim como a avaliação da homogeneidade, a avaliação da estabilidade é também pré-requisito no processo de certificação do MR e tem como objetivo avaliar a estabilidade do mesmo após a sua preparação durante períodos específicos de tempo e temperatura, pois o material preparado pode ser suscetível à degradação com o tempo devido a fenômenos produzidos por: temperatura, luz, oxigênio, umidade, atividade microbiológica etc. Diante desta possibilidade, procedimentos convencionalmente utilizados pela indústria alimentícia para a minimização da atividade microbiana na mesma podem ser empregados na preparação de tais MR (SILVA et al., 2006). Para a produção do MRC proposto neste trabalho foram exploradas a pasteurização (GAVA, 1999) e a irradiação gama (BRASIL, 2001) visando maximizar o prazo de validade dos MRC preparados (CARDOSO, 2008).
Os materiais de referência geralmente são caracterizados baseados na exatidão de todo o processo experimental e desse modo o valor certificado da propriedade representa a melhor estimativa do valor verdadeiro. A incerteza declarada desse valor da propriedade deve levar em consideração todos os efeitos casuais e sistemáticos inerentes ao processo de medição, tão bem como a variabilidade entre as amostras (homogeneidade) e o tempo de validade do material (estabilidade) (INTERNATIONAL ORGANIZATION FOR STANDARDIZATION, 2006).

A matriz tomate foi escolhida para a preparação do MRC uma vez que o programa de monitoramento de hortifrutigranjeiros realizado pela ANVISA (PARA) (BRASIL, 2008) apontou para uma extensiva contaminação daquela verdura com agrotóxicos de diferentes classes e grupos químicos utilizados no Brasil. Adiante, frente ao consumo alimentar no país, a avaliação da contaminação do tomate com resíduos de agrotóxicos assume importância significativa em se tratando de análise de resíduos.

O objetivo principal desse projeto foi estudar as condições mais adequadas para a produção de uma matriz referência certificada utilizando uma matriz natural que foi fortificada com concentrações residuais e conhecida de quatro agrotóxicos $(\gamma-\mathrm{HCH}$, fenitrotiona, clorpirifós e procimidona) seguindo normas nacionais e internacionais de boas práticas.

\section{Material e métodos}

\subsection{Soluções}

Todos os padrões analíticos utilizados apresentam certificado de análise do fabricante com purezas superior a $98 \%$, solventes de diluição grau resíduos de pesticidas e todas as pesagens foram supervisionadas por um segundo analista. A balança analítica utilizada estava calibrada e apresentava-se em conformidade com as normas de qualidade (ASSOCIAÇÃO BRASILEIRA DE NORMAS TÉCNICAS, 2005).

\section{Solução estoque de $\gamma$-HCH e procimidona}

As soluções estoques, individuais, de $100 \mu \mathrm{g} \cdot \mathrm{mL}^{-1}$ foram preparadas por dissolução de cerca de $0,01 \mathrm{~g}$ de $\gamma-\mathrm{HCH}$ e procimidona (Dr. Ehrenstorfer, Alemanha) para $100 \mathrm{~mL}$ de isooctano (Tedia, EUA). As soluções foram armazenadas em freezer $\left(-10 \mathrm{a}-25^{\circ} \mathrm{C}\right)$ até a utilização.

\section{Solução estoque de fenitrotiona}

A solução estoque de $100 \mu \mathrm{g} \cdot \mathrm{mL}^{-1}$ foi preparada por dissolução de cerca de 0,01 g de fenitrotiona (Dr. Ehrenstorfer, Alemanha) para $100 \mathrm{~mL}$ de acetato de etila (Tedia, EUA). As soluções foram armazenadas em freezer $\left(-10 \mathrm{a}-25^{\circ} \mathrm{C}\right)$ até a utilização.

\section{Solução estoque de clorpirifós}

A solução estoque de $100 \mu \mathrm{g} \cdot \mathrm{mL}^{-1}$ foi preparada por dissolução de cerca de 0,01 g de clorpirifós (Riedel-de-Haën, Alemanha) para $100 \mathrm{~mL}$ de acetato de etila (Tedia, EUA). As 
soluções foram armazenadas em freezer $\left(-10 \mathrm{a}-25^{\circ} \mathrm{C}\right)$ até a utilização.

\section{Soluções de fortificação}

As soluções individuais de fortificação na concentração nominal entre 40 e $50 \mu \mathrm{g} \cdot \mathrm{mL}^{-1}$ foram preparadas por diluição de $10 \mathrm{~mL}$ da solução estoque correspondente para $25 \mathrm{~mL}$ de acetona (Tedia, EUA). As concentrações das referidas soluções utilizadas para cada etapa (tratamento por pasteurização e radiação gama) do estudo de homogeneidade estão apresentadas na Tabela 1. As variabilidades demonstradas nas concentrações de fortificação referente às três situações distintas devem-se ao preparo de diferentes soluções estoque. As soluções foram armazenadas em freezer $\left(-10 \mathrm{a}-25^{\circ} \mathrm{C}\right)$ até a utilização. Essas concentrações correspondem à faixa de 0,1 a $0,2 \mathrm{mg}$ de agrotóxico por $\mathrm{kg}$ de amostra.

\subsection{Amostra}

Foram utilizados, neste trabalho, tomates orgânicos provenientes da região serrana do Rio de Janeiro, os quais foram empregados no estudo da produção do MRC, bem como, para o preparo do material de referência nomeado de INCQS-MRC01.

A análise da polpa foi realizada por cromatografia gasosa, acoplada a detector seletivo de massas (CG/DSM), para confirmação da ausência de resíduos de agrotóxicos, e por cromatografia em fase gasosa de alta resolução, acoplada a detector por captura de elétrons (CGAR/DCE), para quantificação dos agrotóxicos estudados utilizando-se um método de multirresíduos.

Primeiramente, com o intuito de avaliar a maneira mais adequada de preparo da amostra, garantindo assim sua homogeneidade, houve a necessidade da realização de um estudo prévio, descritos a seguir. Após o estabelecimento das condições adequadas de preparo da amostra produziu-se o material INCQS-MRC01.

\section{Etapa prévia}

Na etapa prévia deste trabalho utilizou-se aproximadamente $45 \mathrm{~kg}$ de tomate. As amostras foram lavadas, descascadas, e as sementes foram retiradas antes de serem processadas em um liquidificador. Em seguida, passou-se a polpa de tomate processada através de uma peneira com abertura de malha de $0,84 \mathrm{~mm}$, a fim de proporcionar uma redução adicional do particulado da amostra

Tabela 1. Concentrações das soluções de fortificação utilizadas em cada tratamento da amostra.

\begin{tabular}{lccc}
\hline & \multicolumn{3}{c}{ Concentrações das soluções de fortificação $\left(\mu \mathrm{g} \cdot \mathrm{mL}^{-1}\right)$} \\
\hline & $\begin{array}{c}\text { Tratamento por } \\
\text { pasteurização } \\
\text { na etapa prévia }\end{array}$ & $\begin{array}{c}\text { Tratamento por } \\
\text { irradiação gama } \\
\text { na etapa prévia }\end{array}$ & $\begin{array}{c}\text { Tratamento por } \\
\text { irradiação gama do } \\
\text { INCQS-MRC01 }\end{array}$ \\
\hline$\gamma$-HCH & 40,24 & 40,24 & 56,52 \\
Fenitrotiona & 49,96 & 44,36 & 44,36 \\
Clorpirifós & 47,92 & 40,84 & 51,58 \\
Procimidona & 49,66 & 49,66 & 42,73 \\
\hline
\end{tabular}

(ARMISHAW; MILLAR, 2001). O volume de polpa de tomate processado foi homogeneizado com o auxílio de um agitador mecânico e, após 30 minutos, retirou-se cerca de $5 \mathrm{~kg}$ da mesma para servir como branco da amostra e para a determinação do valor do $\mathrm{pH}$. O pH foi medido na polpa processada antes e após a adição dos agrotóxicos. O restante da polpa foi transferida para recipientes de vidro e armazenada sob temperaturas de -10 a $-25^{\circ} \mathrm{C}$ até o momento de fortificação com os agrotóxicos.

\section{Envase do branco da amostra da etapa prévia}

Os cinco quilos do branco da polpa, separados na etapa prévia, foram transferidos para uma batedeira industrial (capacidade para $6 \mathrm{~L}$ ) e foram homogeneizados por 30 minutos. Em seguida, foram envasadas em ampolas de vidro inequivocamente identificadas, as quais foram armazenadas em congelador $(-10$ a $-25^{\circ} \mathrm{C}$ ) após serem seladas. A capacidade de cada ampola é de $30 \mathrm{~mL}$, o que corresponde à massa de 14 a $20 \mathrm{~g}$ de polpa por ampola. Durante a transferência do volume para as ampolas de vidro, a agitação da batelada de amostra não foi interrompida e a velocidade da hélice da batedeira foi ajustada de modo a proporcionar agitação sem provocar respingos do líquido.

\section{Fortificação da polpa de tomate na etapa prévia}

A elaboração desta etapa foi realizada em dois momentos distintos, uma parte do material fortificado foi reservada para o estudo da estabilidade após tratamento por pasteurização e outra parte para tratamento por irradiação gama, ambos após confirmação da homogeneidade. Diante do exposto, a parte apresentada a seguir foi seguida para cada um dos referidos tratamentos.

Aproximadamente 12 a $14 \mathrm{~kg}$ da polpa de tomate preparada na etapa prévia foram retirados da refrigeração $\left(-10 \mathrm{a}-25^{\circ} \mathrm{C}\right) \mathrm{e}$ utilizados para a fortificação do MRC. Esse volume foi transferido para uma batedeira industrial (capacidade para $20 \mathrm{~L}$ ), sendo mantido sob agitação por 30 minutos para homogeneização. Em seguida, cerca de $700 \mathrm{~g}$ deste material foi transferido para liquidificador, de copo de vidro, para que as quatro soluções individuais de fortificação, indicadas na Tabela 1, fossem então, lentamente adicionadas. Após a adição das mesmas, o material foi mantido à velocidade mínima por 1 hora e seu $\mathrm{pH}$ foi medido. Logo após essa etapa, as cerca de $700 \mathrm{~g}$ de polpa fortificada foram adicionados à massa restante na batedeira industrial (capacidade para $20 \mathrm{~L}$ ), para homogeneização por mais três horas, a fim de proporcionar uma melhor interação da polpa de tomate com os padrões de agrotóxicos. Em seguida, o material foi transferido para ampolas de vidro inequivocamente identificadas, as quais foram armazenadas em congelador $\left(-10 \mathrm{a}-25^{\circ} \mathrm{C}\right)$ após serem seladas, até o devido tratamento (por pasteurização ou por irradiação gama) visando à conservação da amostra. Quatrocentos e vinte unidades de ampolas contendo o material fortificado foram produzidas, sendo que vinte destinaram-se para os testes de homogeneidade e as restantes foram reservadas a avaliação em relação à estabilidade, sob condições pré-estabelecidas (CARDOSO, 2008). A capacidade de cada ampola é de $30 \mathrm{~mL}$, o que corresponde à massa de $14 \mathrm{a} 20 \mathrm{~g}$ de material por ampola. Durante a transferência do volume necessário para as ampolas de vidro, a agitação da batelada de amostra não foi interrompida 
e a velocidade da hélice da batedeira foi ajustada de modo a proporcionar agitação sem provocar respingos do líquido.

\section{Preparo do material INCQS-MRC01}

Quinze quilos de tomate foram utilizados para a produção da matriz candidata a tornar-se material de referência, conforme as mesmas condições apresentadas nas etapas prévias.

A quantidade de ampolas do material INCQS-MRC01, contendo polpa de tomate fortificada totalizou 220 unidades e 100 ampolas correspondendo ao branco da amostra.

\section{Tratamento por irradiação gama}

As ampolas contendo amostras fortificadas e brancos da matriz foram irradiadas com dose de $2 \mathrm{kGy}$ no irradiador de pesquisa com fonte de césio-137 (com atividade atual de $46 \mathrm{kCi}$ ) localizado no Centro Tecnológico do Exército (VITAL et al., 2000; CARDOSO et al., 2008).

\section{Tratamento por pasteurização}

As ampolas contendo amostras fortificadas e brancos da matriz foram imersas em banho a temperatura de $90{ }^{\circ} \mathrm{C}$ por 4 minutos, seguido de banho de gelo (CARDOSO, 2008).

\subsection{Avaliação do pH}

As medidas de $\mathrm{pH}$ ocorreram pelo emprego de medidor de pH (Mettler Toledo) modelo MP 220.

\subsection{Procedimento de extração}

Para quantificação dos quatro analitos na polpa de tomate empregou-se um procedimento validado previamente por Cardoso (2008). Resumidamente, $15 \mathrm{~g}$ da amostra são extraídas com $30 \mathrm{~mL}$ de acetona e, em seguida, com $60 \mathrm{~mL}$ de uma mistura diclorometano:éter de petróleo $(1: 1, \mathrm{v}: \mathrm{v})$. O recipiente é levado à centrifugação e, em seguida a fase orgânica é filtrada em sulfato de sódio. Desse volume coletado, $1 \mathrm{~mL}$ é evaporado e dissolvido em $1 \mathrm{~mL}$ de isooctano para análise por CGAR/DCE. Para análise por CG/DSM, foram retirados $25 \mathrm{~mL}$ do mesmo extrato filtrado, evaporado em banho de aquecimento até no máximo $70^{\circ} \mathrm{C}$, dissolvido em $1 \mathrm{~mL}$ de uma mistura de padrão interno em acetato de etila e $2 \mu \mathrm{l}$ do extrato foram injetados no cromatógrafo.

\subsection{Instrumentação e condições cromatográficas}

Nos experimentos foram utilizados os seguintes equipamentos com respectivos ajustes e procedimentos:

Cromatógrafo a gás HP 6890 (Agilent), devidamente qualificado, equipado com detector por captura de elétrons. Temperatura do injetor e detector de 210 e $300^{\circ} \mathrm{C}$, respectivamente. Coluna HP 5MS ( $30 \mathrm{~m} \times 0,25 \mathrm{~mm} \times 0,25 \mu \mathrm{m})$. Programação do gradiente de temperatura do forno: $80^{\circ} \mathrm{C}$ (0 minutos) ${ }^{\oplus 30}{ }^{\circ} \mathrm{C} /$ minutos $180{ }^{\circ} \mathrm{C}$ (8 minutos) ${ }^{\oplus 2} 2^{\circ} \mathrm{C} /$ minutos $200{ }^{\circ} \mathrm{C}$ (5 minutos) ${ }^{\circledR 6} 6^{\circ} \mathrm{C} /$ minutos $280^{\circ} \mathrm{C}(5$ minutos). Fluxo de gás carreador
$($ Hélio $)=1,2 \mathrm{~mL} \cdot \mathrm{min}^{-1}$, injeção "splitless" $=0,75$ minutos, volume injetado $=1,0 \mu \mathrm{L}$.

Cromatógrafo a gás acoplado a detector seletivo de massas (Agilent 6890/5973). Temperatura do injetor de $230^{\circ} \mathrm{C}$. Coluna HP 5 MS $(30 \mathrm{~m} \times 0,25 \mathrm{~mm} \times 0,25 \mu \mathrm{m})$ com programação de gradiente de temperaturas: $60{ }^{\circ} \mathrm{C}(2,50$ minutos $){ }^{\oplus 10^{\circ} \mathrm{C} / \text { minutos }}$ $180^{\circ} \mathrm{C}(1$ minuto $){ }^{\oplus{ }^{\circ} \mathrm{C} / \text { minutos }} 215^{\circ} \mathrm{C}(1$ minuto $){ }^{@ 10^{\circ} \mathrm{C} / \text { minutos }} 280^{\circ} \mathrm{C}$ (15 minutos). Fluxo de gás carreador (Hélio) $=1,0 \mathrm{~mL} \cdot$ minutos $^{-1}$, injeção "pulsed splitless" $=0,75$ minutos. Temperatura da linha de transferência de $280^{\circ} \mathrm{C}$. Varredura de íons na faixa de 90 a $450 \mathrm{~mm}$.

\subsection{Teste de homogeneidade}

Para avaliação da homogeneidade dos lotes preparados na etapa prévia, bem como do material INCQS-MRC01, 10 ampolas foram aleatoriamente selecionadas antes e após aplicação do tratamento visando garantir a estabilidade do mesmo, usando-se o programa Windows/Excel ${ }^{\circ}$ antes do início do envasamento, de modo a facilitar a identificação dos frascos destinados ao teste. Os agrotóxicos foram extraídos da amostra de acordo com o método multirresíduos apresentado no item $2.4 \mathrm{e}$ foram analisadas por CGAR/DCE conforme condições indicadas no item 2.5. Cada ampola lacrada originou duas alíquotas resultantes do mesmo extrato da amostra, denominadas de replicatas A e B. O teste de homogeneidade foi realizado segundo recomendação da ISO GUIDE 35 (van der VEEN; LISINGER; PAUWLES, 2001; INTERNATIONAL ORGANIZATION FOR STANDARDIZATION, 2006). Os dados obtidos foram avaliados estatisticamente, utilizando a análise de variâncias (ANOVA) com fator único (INTERNATIONAL ORGANIZATION FOR STANDARDIZATION, 2006), a partir da qual se pode verificar se a variação na composição das amostras distribuídas nos recipientes foi suficientemente pequena para o objetivo proposto.

\section{Incertezas inerentes à homogeneidade}

A parcela da incerteza inerente à homogeneidade $\left(u_{\text {homogeneidade }}\right)$ do material que compõe o lote INCQS-MRC01 preparado foi obtida a partir dos mesmos dados gerados pela ANOVA com fator único (van der VEEN; LISINGER; PAUWLES, 2001; INTERNATIONAL ORGANIZATION FOR STANDARDIZATION, 2006).

\subsection{Estudo interlaboratorial para caracterização do material INCQS-MRCO1}

Sete laboratórios foram convidados a participar da etapa de certificação do material INCQS-MRC01. Os laboratórios foram escolhidos por apresentarem a certificação segundo a norma ISO/ IEC 17025 (ASSOCIAÇÃO BRASILEIRA DE NORMAS TÉCNICAS, 2005) e/ou por terem demonstrado performances satisfatórias em estudos de proficiência organizados pelo INCQS.

Foram enviados aos laboratórios dois conjuntos de amostras, um com seis ampolas de vidro seladas, contendo, cada uma, a polpa de tomate fortificada com resíduos dos quatro agrotóxicos alvos da produção e outro com duas ampolas de vidro seladas, contendo, cada uma, a polpa de tomate branco. Cada laboratório 
recebeu instruções de como proceder ao preparo das ampolas para amostragem devendo realizar a análise preparando 3 porções individuais (replicatas) da amostra de purê de tomate com resíduos de quatro agrotóxicos (ampolas identificadas numericamente) e pelo menos 1 amostra Branco empregando para tal, o método de rotina do laboratório.

\section{Tratamento estatístico dos dados do estudo interlaboratorial}

O procedimento experimental para análise de dados segue indicação do item 10.5.2 da international Organization for Standardization (2006) (van der VEEN et al., 2001), a qual indica que a média das médias $(\overline{\bar{x}})$ dos laboratórios participantes é a melhor escolha para ser o valor de propriedade do material avaliado no estudo interlaboratorial. Para avaliação dos valores discrepantes aplicou-se os testes de Cochran e de Grubbs e para cálculo dos valores das incertezas associadas às médias dos laboratórios foram empregadas as Equações 1 e 2 (INTERNATIONAL ORGANIZATION FOR STANDARDIZATION,2006).

$$
\begin{aligned}
& u_{\text {carac }}=\frac{s}{\sqrt{p}} \\
& s=\sqrt{\frac{1}{p-1} \sum_{i=1}^{p}\left(\overline{x_{1}}-\bar{x}\right)^{2}}
\end{aligned}
$$

Onde $p$ representa o número de laboratórios participantes no estudo, $s$ é o desvio padrão obtido através da Equação 2, $(\bar{x})$ é a média de cada laboratório, $(\bar{x})$ indica a média das médias de todos os resultados, $\mathrm{e}\left(\overline{x_{1}}-\overline{\bar{x}}\right)^{2}$ representa a soma quadrática das diferenças dos valores médios reportados pelos participantes.

\section{Certificação do material INCQS-MRC01 - atribuição dos valores de propriedade ao MR}

Para o cálculo dos valores de propriedades de cada agrotóxico no material de referência produzido utilizou-se a média ponderada dos dois valores independentes: resultados do laboratório provedor e resultados do estudo interlaboratorial. As estimativas de incertezas foram obtidas por combinação, de acordo com o item 10.8.3, da International Organization for Standardization (2006), das incertezas padrão ponderadas dos referidos valores independentes, acrescidas das incertezas padrão associadas à homogeneidade e a estabilidade conforme demonstrado na Equação 3.

$$
u_{c}^{2}=u_{\mathrm{hom}}^{2}+u_{e s t}^{2}+\sum_{i=1}^{n} w_{i}^{2} u_{i}^{2}
$$

Onde $u_{\mathrm{i}}$ indica a incerteza padrão dos valores de propriedades contribuintes, $w_{\mathrm{i}}$ é o peso dos valores contribuintes $(1 / 2), u_{\mathrm{hom}} \mathrm{e}$ a incerteza padrão associada a homogeneidade e $u_{\text {est }}$ representa a incerteza padrão associada a estabilidade.

\section{Resultados e discussão}

Olote preparado na etapa prévia permitiu investigar o processo de produção e identificar problemas inerentes ao mesmo, bem como avaliar previamente sua estabilidade durante condições de tempo e de temperatura pré-determinados. Os tratamentos utilizados para garantir a integridade da amostra após ter sua homogeneidade confirmada foram: pasteurização e irradiação gama, os quais foram descritos detalhadamente por Cardoso (2008).

As concentrações dos agrotóxicos na matriz a ser transformada em material de referência foram definidas de modo a garantir que os níveis de concentração resultantes dos analitos (faixa de concentração entre 0,1 e 0,2 mg. $\mathrm{kg}^{-1}$ ) pudessem ser detectados em todos os laboratórios envolvidos na pesquisa.

Após a retirada da casca e sementes, o volume final da polpa ficou reduzido a aproximadamente 70 a $80 \%$ de seu volume inicial. A opção pela remoção da casca e das sementes teve como objetivo principal buscar uniformizar as dimensões do particulado de polpa de tomate processada sem a possível interferência de pequenos fragmentos oriundos dessas partes do fruto que poderiam vir a prejudicar o grau de homogeneidade esperado para o lote produzido (ARMISHAW; MILLAR, 2001). Em seguida, o material foi testado para verificação de ausência de resíduos de agrotóxicos por CGAR/ DCE e confirmação por CG/DSM, conforme apresentado por Cardoso (2008), o qual, não apresentou interferentes ou resíduos das substâncias estudadas.

Sendo o tomate um fruto que apresenta alto teor de água, consequentemente, um determinado grau de heterogeneidade é esperado no processo de trituração desse material devido à possibilidade de formação de duas fases (água e precipitado). Sendo assim, foi imprescindível manter a agitação da batelada de amostra durante a transferência do volume para as ampolas.

O valor do $\mathrm{pH}$ na polpa foi levado em consideração, pois deve ser ácido o suficiente para garantir o longo tempo de estocagem após o acondicionamento nos recipientes de vidro, correspondendo a valores de 4,0 e 4,1 respectivamente antes e após a adição das soluções de fortificação, indicando pequena alteração com adição das mesmas. Valores maiores que 4,4 não são desejados, pois propiciariam o crescimento de organismos microbiológicos favorecendo condições para degradação dos analitos (ARMISHAW; MILLAR, 2001).

Os lotes contendo polpa de tomate, preparados com o propósito de avaliar a estabilidade dos agrotóxicos após confirmação da homogeneidade, foram submetidos aos tratamentos por esterilização térmica ou por radiação gama para ser estudada durante o período de um ano. Os resultados obtidos após este tempo são apresentados na íntegra por Cardoso (2008). Os valores das concentrações encontradas para cada agrotóxico na amostra homogeneizada para o estudo da estabilidade após pasteurização e após irradiação gama são indicados nas Tabelas $2 \mathrm{e}$ 3 , respectivamente, bem como, os resultados das análises de variância gerados a partir de 10 ampolas pelo software Microsoft Windows/Excel ${ }^{\oplus}$.

Usando-se o modelo ANOVA fator único (INTERNATIONAL ORGANIZATION FOR STANDARDIZATION, 2006), como ferramenta estatística para avaliação das homogeneidades, a qual apresenta como fontes de variação, as diferenças entre as amostras (linhas) e dentro das amostras (colunas), o teste F indica se os resultados caracterizam a mesma como significantes ou não. Quando $\mathrm{F}_{\text {calculado }}<\mathrm{F}_{\text {critico, (a }=5 \%)}$ aceita-se que há homogeneidade entre as amostras. $\mathrm{O}$ valor de $\mathrm{P}$ indica em qual nível o valor de $\mathrm{F}_{\text {calculado }}$ foi observado. 
Tabela 2. Concentrações médias e desvios padrão (DP) calculados na determinação do $\gamma$-HCH, fenitrotiona, clorpirifós e procimidona na polpa de tomate preparada na etapa prévia, antes e após tratamento por pasteurização e os resultados da homogeneidade das amostras gerada através da análise de variância por fator único.

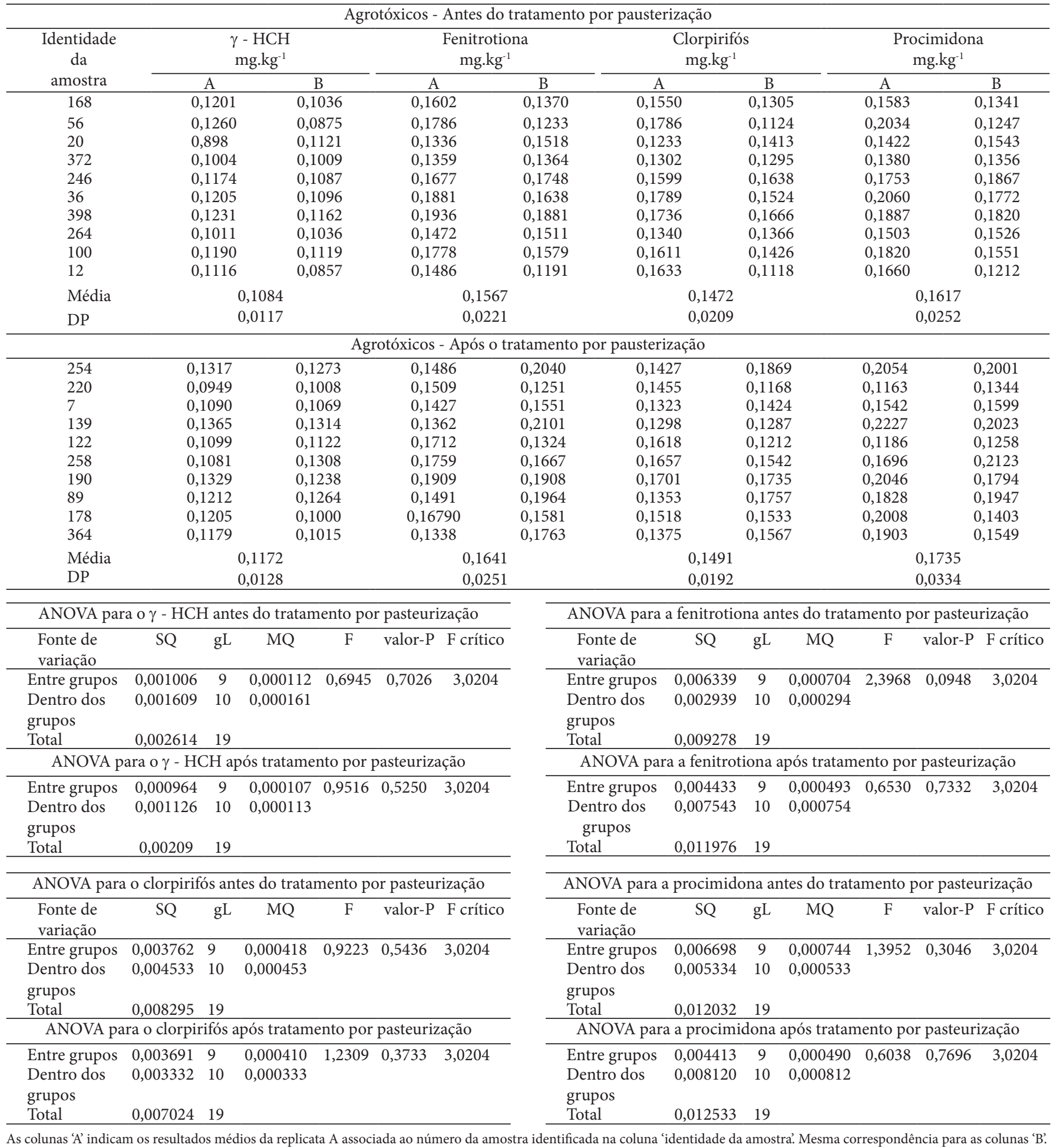

Para os agrotóxicos citados nas Tabelas 2 e 3, o teste F confirmou não haver diferenças significativas entre os resultados, com $\mathrm{F}_{\text {calculado }}<\mathrm{F}_{\text {crítico }}$, e, portanto, estando a amostra homogênea em relação aos agrotóxicos nela presentes antes e após os tratamentos específicos.
Os resultados encontrados para os quatro agrotóxicos bem como o valor do $\mathrm{pH}$, foram satisfatórios em relação à homogeneidade da amostra, indicando que o particulado constante da matriz após utilização da peneira, o uso da acetona como solvente de diluição da solução de fortificação 
Tabela 3. Concentrações médias e desvios padrão (DP) calculados na determinação do $\gamma$-HCH, fenitrotiona, clorpirifós e procimidona na polpa de tomate preparada na etapa prévia, antes e após tratamento por radiação gama e os resultados da homogeneidade das amostras gerada através da análise de variância por fator único.

\begin{tabular}{|c|c|c|c|c|c|c|c|c|c|c|c|c|c|c|}
\hline \multicolumn{15}{|c|}{ Agrotóxicos - Antes do tratamento por radiação gama } \\
\hline \multirow{2}{*}{$\begin{array}{c}\text { Identidade } \\
\text { da } \\
\text { amostra }\end{array}$} & \multicolumn{4}{|c|}{$\begin{array}{c}\gamma-\mathrm{HCH} \\
\text { mg.kg-1 }\end{array}$} & \multicolumn{3}{|c|}{$\begin{array}{c}\text { Fenitrotiona } \\
\text { mg.kg-1 }\end{array}$} & \multicolumn{4}{|c|}{$\begin{array}{c}\text { Clorpirifós } \\
\text { mg.kg-1 }\end{array}$} & \multicolumn{3}{|c|}{$\begin{array}{c}\text { Procimidona } \\
\text { mg.kg-1 }\end{array}$} \\
\hline & \multicolumn{2}{|c|}{ A } & \multicolumn{2}{|c|}{ B } & \multicolumn{2}{|c|}{ A } & B & \multicolumn{2}{|c|}{ A } & \multicolumn{2}{|l|}{ B } & A & & B \\
\hline 19 & \multirow{2}{*}{\multicolumn{2}{|c|}{$\begin{array}{l}0,1839 \\
0,1850\end{array}$}} & 0,17 & & 0,20 & & 0,1975 & 0,17 & & 0,187 & & 0,2490 & & 0,2426 \\
\hline 26 & & & 0,17 & & 0,22 & & 0,2253 & 0,17 & & 0,176 & & 0,2410 & & 0,2399 \\
\hline 35 & & 707 & 0,18 & & 0,21 & & 0,2138 & 0,17 & & 0,187 & & 0,2452 & & 0,2488 \\
\hline 47 & & 864 & 0,17 & & 0,20 & & 0,2054 & 0,17 & & 0,162 & & 0,2408 & & 0,2255 \\
\hline 96 & & 823 & 0,17 & & 0,20 & & 0,2163 & 0,18 & & 0,198 & & 0,2294 & & 0,2277 \\
\hline 153 & & 845 & 0,19 & & 0,21 & & 0,2163 & 0,16 & & 0,178 & & 0,2303 & & 0,2401 \\
\hline 264 & & 721 & 0,18 & & 0,21 & & 0,2236 & 0,19 & & 0,199 & & 0,2287 & & 0,2358 \\
\hline 298 & & 841 & 0,17 & & 0,21 & & 0,2256 & 0,18 & & 0,163 & & 0,2470 & & 0,2378 \\
\hline 310 & & 739 & 0,18 & & 0,22 & & 0,2224 & 0,17 & & 0,174 & & 0,2383 & & 0,2448 \\
\hline Média & & & 0,1809 & & & 0,2129 & & & 0,1808 & & & & 0,2382 & \\
\hline DP & & & 0,0066 & & & 0,0111 & & & 0,0107 & & & & 0,0072 & \\
\hline & & & & Agro & otóxicos - & - Após o tr & atamentc & to por radiaçãc & gama & & & & & \\
\hline 5 & & 650 & 0,16 & & 0,20 & & 0,1897 & 0,18 & & 0,170 & & 0,2403 & & 0,2266 \\
\hline 13 & & 601 & 0,15 & & 0,21 & & 0,2100 & 0,18 & & 0,177 & & 0,2378 & & 0,2443 \\
\hline 77 & & 649 & 0,15 & & 0,21 & & 0,2259 & 0,19 & & 0,192 & & 0,2458 & & 0,2446 \\
\hline 85 & & 633 & 0,15 & & 0,20 & & 0,2051 & 0,18 & & 0,193 & & 0,2368 & & 0,2489 \\
\hline 122 & & 462 & 0,16 & & 0,20 & & 0,2133 & 0,17 & & 0,170 & & 0,2288 & & 0,2301 \\
\hline 144 & & 596 & 0,15 & & 0,19 & & 0,2173 & 0,16 & & 0,183 & & 0,2203 & & 0,2439 \\
\hline 158 & & 593 & 0,15 & & 0,21 & & 0,2106 & 0,17 & & 0,170 & & 0,2300 & & 0,2273 \\
\hline 209 & & 629 & 0,16 & & 0,20 & & 0,2089 & 0,18 & & 0,190 & & 0,2371 & & 0,2396 \\
\hline 265 & & 520 & 0,15 & & 0,20 & & 0,2106 & 0,19 & & 0,171 & & 0,2357 & & 0,2399 \\
\hline 355 & & 573 & 0,16 & 639 & 0,20 & & 0,2270 & 0,17 & & 0,187 & & 0,2377 & & 0,2476 \\
\hline Média & & & 0,1589 & & & 0,2099 & & & 0,1800 & & & & 0,2371 & \\
\hline DP & & & 0,0053 & & & 0,0085 & & & 0,0098 & & & & 0,0078 & \\
\hline $\mathrm{ANO}$ & VA para o & $-\mathrm{H}$ & $\mathrm{CH}$ antes da & radiaçã & o gama & & & ANOI & A para a fe & enitrot & iona antes c & da radiaç & ção gama & \\
\hline $\begin{array}{l}\text { Fonte de } \\
\text { variação }\end{array}$ & SQ & $\mathrm{gL}$ & MQ & $\mathrm{F}$ & valor-P & F crítico & & $\begin{array}{l}\text { Fonte de } \\
\text { variação }\end{array}$ & SQ & gL & MQ & $\mathrm{F}$ & valor-P & F crítico \\
\hline Entre grupos & 0,000216 & 9 & 0,000024 & 0,3854 & 0,9165 & 3,0204 & & Entre grupos & 0,001632 & 9 & 0,0001813 & 2,5861 & 0,0775 & 3,0204 \\
\hline $\begin{array}{l}\text { Dentro dos } \\
\text { grupos }\end{array}$ & 0,000621 & 10 & 0,000062 & & & & & $\begin{array}{l}\text { Dentro dos } \\
\text { grupos }\end{array}$ & 0,000701 & 10 & 0,0000701 & & & \\
\hline Total & 0,000837 & 19 & & & & & & Total & 0,002333 & 19 & & & & \\
\hline $\mathrm{ANC}$ & DVA para o & $\gamma-\mathrm{H}$ & $\mathrm{ICH}$ após a 1 & radiação & gama & & & ANC & VA para a & fenitr & otiona após & s radiaçãc & o gama & \\
\hline Entre grupos & 0,000283 & 9 & 0,000031 & 1,2874 & 0,3480 & 3,0204 & & Entre grupos & 0,000901 & 9 & 0,000100 & 2,1008 & 0,1315 & 3,0204 \\
\hline $\begin{array}{l}\text { Dentro dos } \\
\text { grupos }\end{array}$ & 0,000245 & 10 & 0,000024 & & & & & $\begin{array}{l}\text { Dentro dos } \\
\text { grupos }\end{array}$ & 0,000477 & 10 & 0,000048 & & & \\
\hline Total & 0,000528 & 19 & & & & & & Total & 0,001378 & 19 & & & & \\
\hline ANO & VA para o c & orpi & ifós antes $\mathrm{d}$ & a radiaçã & ão gama & & & ANOV & A para a pr & rocimi & dona antes & da radiac & ção gama & \\
\hline $\begin{array}{l}\text { Fonte de } \\
\text { variação }\end{array}$ & SQ & $\mathrm{gL}$ & MQ & $\mathrm{F}$ & valor-P & F crítico & & $\begin{array}{l}\text { Fonte de } \\
\text { variação }\end{array}$ & SQ & $\mathrm{gL}$ & MQ & $\mathrm{F}$ & valor-P & F crítico \\
\hline Entre grupos & 0,001545 & 9 & 0,0001717 & 2,7870 & 0,0630 & 3,0204 & & Entre grupos & 0,000678 & 9 & 0,000075 & 2,5693 & 0,0789 & 3,0204 \\
\hline $\begin{array}{l}\text { Dentro dos } \\
\text { grupos }\end{array}$ & 0,000616 & 10 & 0,000062 & & & & & $\begin{array}{l}\text { Dentro dos } \\
\text { grupos }\end{array}$ & 0,000293 & 10 & 0,000029 & & & \\
\hline Total & 0,002162 & 19 & & & & & & Total & 0,000972 & 19 & & & & \\
\hline $\mathrm{ANC}$ & DVA para o & clor & pirifós após & radiação & gama & & & $\mathrm{ANO}$ & VA para a $p$ & procin & nidona após & s radiaçã & fo gama & \\
\hline Entre grupos & 0,001284 & 9 & 0,000143 & 2,7159 & 0,0677 & 3,0204 & & Entre grupos & 0,000630 & 9 & 0,000070 & 1,3159 & 0,3360 & 3,0204 \\
\hline $\begin{array}{l}\text { Dentro dos } \\
\text { grupos }\end{array}$ & 0,000525 & 10 & 0,000053 & & & & & $\begin{array}{l}\text { Dentro dos } \\
\text { grupos }\end{array}$ & 0,000532 & 10 & 0,000053 & & & \\
\hline Total & 0,001809 & 19 & & & & & & Total & 0,001162 & 19 & & & & \\
\hline
\end{tabular}

e o tempo de homogeneização de 3 horas foram satisfatórios para a produção da matriz tomate como um material de referência contendo $\gamma$ - $\mathrm{HCH}$, fenitrotiona, clorpirifós e procimidona.
Diante dos resultados prévios não ocorreram dificuldades para o preparo do lote do material INCQS-MRC01. Para este lote preparado o valor do $\mathrm{pH}$ antes da adição das soluções de fortificação e antes do envasamento nas ampolas foi de 4,0. 
Tabela 4. Concentrações médias, desvios padrão (DP) calculados na determinação do $\gamma$-HCH, fenitrotiona, clorpirifós e procimidona na polpa de tomate antes e após tratamento por radiação gama para avaliação da homogeneidade do material INCQS-MRC01 e os resultados da homogeneidade das amostras gerada através da análise de variância por fator único.

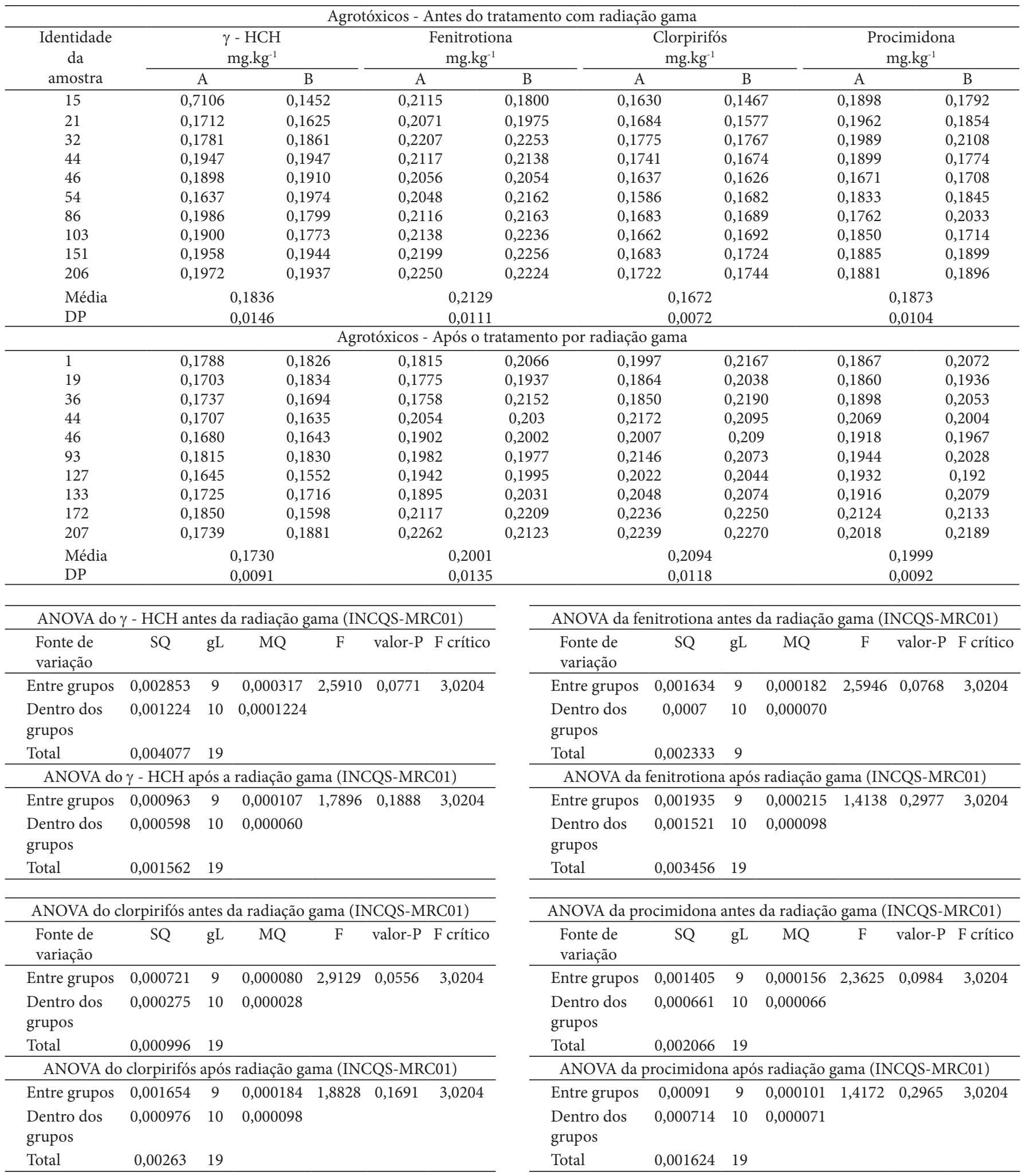

As colunas 'A' indicam os resultados médios da replicata A associada ao número da amostra identificada na coluna 'identidade da amostra'. Mesma correspondência para as colunas 'B'. 
A verificação prévia da homogeneidade foi confirmada com os dados gerados a partir de 10 ampolas, indicando que as amostras encontravam-se em estado apropriado e poderiam então ser expostas à radiação gama.

Após a irradiação, a homogeneidade das amostras e sua incerteza foram avaliadas com os resultados obtidos relativos ao lote restante, subtraído de 10 amostras. Na Tabela 4 são apresentadas as concentrações médias e desvios padrão obtidos para cada unidade de ampola testada antes e após serem irradiadas e os respectivos resultados gerados através das análises de variância por fator único. Pode-se observar também neste lote que o teste $\mathrm{F}$ demonstrou que a variabilidade entre as amostras foram insignificativas.

A incerteza da homogeneidade é função dos valores da média quadrática $(\mathrm{MQ})$ entre as ampolas $\left(\mathrm{MQ}_{\text {entre }}\right)$ e dentro das ampolas $\left(\mathrm{MQ}_{\text {dentro }}\right)$, a qual também é fornecida pelo teste de análise de variância (Tabelas 2 a 4). Quando o valor de MQ entre as ampolas $\left(\mathrm{MQ}_{\text {entre }}\right)$ for maior que dentro das mesmas $\left(\mathrm{MQ}_{\text {dentro }}\right)$, a incerteza padrão devido à não homogeneidade $\left(u_{\text {homogeneidade }}\right.$ ou $\left.u_{\mathrm{bb}}\right)$ é equivalente ao desvio padrão $\left(\mathrm{S}_{\mathrm{bb}}\right)$ entre as ampolas, podendo ser estimada através da Equação 4, com $\mathrm{n}=$ número de replicatas das ampolas. Neste estudo, as ampolas continham quantidade referente a apenas uma pesagem de amostra, resultando em $\mathrm{n}=1$ replicata por ampola. Desse modo, nos casos em que a sub-amostragem é inviável, ou seja, quando não é possível se efetuar duas pesagens do mesmo recipiente, a variabilidade dentro das ampolas é inferida da reprodutibilidade (replicatas A e B) da mesma unidade sob medição (INTERNATIONAL ORGANIZATION FOR STANDARDIZATION, 2006; van der VEEN; LISINGER; PAUWLES, 2001). Para o cálculo da incerteza expandida $\left(U_{\text {hom }}\right)$ considera-se o fator de abrangência 2, para o grau de confiança de $95 \%$.

$u_{b b}=S_{b b}=\sqrt{\frac{M Q_{\text {entre }}-M Q_{\text {dentro }}}{n}}$

Na Tabela 5, são apresentados os valores das incertezas (variâncias) referentes à homogeneidade do material INCQSMRC01, obtidos após irradiação, calculados usando-se a Equação 4 para os agrotóxicos estudados.

Após realização do teste de homogeneidade, foi conduzido um programa interlaboratorial para a execução da etapa de certificação do lote de polpa de tomate preparado, INCQSMRC01. Embora os sete laboratórios convidados a participar desta etapa tenham recebido o material, apenas cinco enviaram os resultados. Nem todos os laboratórios determinaram todos os agrotóxicos solicitados. Na Tabela 6 são apresentados os resultados reportados pelos colaboradores. Cada resultado é a média das análises em triplicatas, a partir da qual o valor da média de todos os laboratórios $(\underset{x}{x})$ foi adotada como resultado do estudo interlaboratorial.

Os resultados encontrados pelos laboratórios colaboradores estão de acordo com os resultados determinados pelo laboratório produtor através do teste de homogeneidade. Cada participante enviou juntamente com as concentrações encontradas informações sobre os métodos analíticos empregados para as análises.

De posse dos resultados enviados foi possível então determinar os valores de propriedades do material INCQSMRC01. Na Tabela 7 são apresentados os valores de propriedades que contribuíram para a certificação do material INCQSMRC01 e incertezas associadas. A incerteza inerente ao valor certificado foi calculada através da Equação 4. Os valores de incerteza atribuídos ao resultado do laboratório provedor do material, bem como, à característica de estabilidade do material foram estudados por Cardoso (2008).

Tabela 5. Valores das incertezas referentes à homogeneidade do material de referência MRC01 para os agrotóxicos estudados.

\begin{tabular}{lccc}
\hline Agrotóxico & $\begin{array}{c}\text { Resultado médio } \\
\left(\mathrm{mg}^{\left.-k^{-1}\right)}\right.\end{array}$ & $\begin{array}{c}\text { Incerteza padrão } \\
\left(\mathrm{Sbb}=\boldsymbol{u}_{\text {homogeneidade }}\right) \\
\left(\mathrm{mg}^{\mathrm{k}} \mathrm{kg}^{-1}\right)\end{array}$ & $\begin{array}{c}\mathrm{U}_{\text {hom }}(\mathrm{k}=2)^{1} \\
\left(\mathrm{mg}^{1} \mathrm{~kg}^{-1}\right)\end{array}$ \\
\hline$\gamma$-HCH & 0,1730 & 0,0069 & 0,0138 \\
Fenitrotiona & 0,2001 & 0,0079 & 0,0158 \\
Clorpirifós & 0,2094 & 0,0093 & 0,0186 \\
Procimidona & 0,1999 & 0,0055 & 0,0110 \\
\hline O cálculo da estimativa da incerteza expandida utilizou o fator de abrangência, $\mathrm{k}=2$.
\end{tabular}

Tabela 6. Resultados reportados pelos participantes do estudo interlaboratorial e tratamento estatístico dos dados para atribuição do valor referência com incerteza associada. Cada resultado individual representa a média das análises efetuadas em triplicatas.

\begin{tabular}{ccccc}
\hline Laboratório & $\begin{array}{c}\gamma-\mathrm{HCH} \\
\left(\mathrm{mg}^{\mathrm{kg}} \mathrm{kg}^{-1}\right)\end{array}$ & $\begin{array}{c}\text { Fenitrotiona } \\
\left.\left(\mathrm{mg}^{\mathrm{kg}}\right)^{-1}\right)\end{array}$ & $\begin{array}{c}\text { Clorpirifós } \\
\left(\mathrm{mg} \mathrm{kg}^{-1}\right)\end{array}$ & $\begin{array}{c}\text { Procimidona } \\
\left(\mathrm{mg}^{-k^{-1}}\right)\end{array}$ \\
\hline $\mathrm{A}$ & 0,195 & 0,188 & 0,292 & 0,189 \\
$\mathrm{~B}$ & 0,175 & 0,144 & 0,160 & 0,155 \\
$\mathrm{C}$ & 0,263 & 0,233 & 0,320 & - \\
$\mathrm{D}$ & 0,180 & 0,167 & 0,193 & 0,117 \\
$\mathrm{E}$ & 0,228 & - & - & - \\
$\bar{x}$ & 0,208 & 0,183 & 0,241 & 0,153 \\
$\mathrm{~S}$ & 0,0374 & 0,0387 & 0,0766 & 0,0361 \\
$u_{\text {carac }}$ & 0,0165 & 0,0191 & 0,03829 & 0,0209 \\
$\mathrm{U}(\mathrm{k}=2,95 \%)^{1}$ & 0,0330 & 0,0382 & 0,07659 & 0,0417 \\
\hline $\mathrm{O}$ cálculo da estimativa da incerteza expandida utilizou o fator de abrangência, $\mathrm{k}=2$.
\end{tabular}

Tabela 7. Valores atribuídos as propriedades do material INCQS-MRC01 e suas incertezas.

\begin{tabular}{|c|c|c|c|c|c|}
\hline Agrotóxico & $\begin{array}{l}\text { Resultado Lab. provedor } \\
\qquad\left(\mathrm{mg}^{\mathrm{kg}} \mathrm{kg}^{-1}\right)\end{array}$ & $\begin{array}{l}\text { Resultado Interlaboratorial } \\
\left(\mathrm{mg} \cdot \mathrm{kg}^{-1}\right)\end{array}$ & $\begin{array}{c}\mathrm{U}_{\text {hom }}{ }^{1} \\
\left(\mathrm{mg}^{\left.-k g^{-1}\right)}\right.\end{array}$ & $\begin{array}{c}\mathrm{U}_{\text {est }}{ }^{1} \\
\left(\mathrm{mg} \cdot \mathrm{kg}^{-1}\right)\end{array}$ & $\begin{array}{l}\text { Valor certificado } \\
\left(\mathrm{mg} \cdot \mathrm{kg}^{-1}\right)\end{array}$ \\
\hline$\gamma-\mathrm{HCH}$ & $0,1730 \pm 0,070$ & $0,208 \pm 0,033$ & 0,0138 & 0,0176 & $0,191 \pm 0,047$ \\
\hline fenitrotiona & $0,2001 \pm 0,110$ & $0,183 \pm 0,038$ & 0,0158 & 0,0320 & $0,192 \pm 0,068$ \\
\hline clorpirifós & $0,2094 \pm 0,109$ & $0,241 \pm 0,077$ & 0,0186 & 0,0316 & $0,225 \pm 0,076$ \\
\hline procimidona & $0,1999 \pm 0,088$ & $0,153 \pm 0,042$ & 0,0110 & 0,0090 & $0,177 \pm 0,051$ \\
\hline
\end{tabular}

$\mathrm{O}$ cálculo da estimativa da incerteza expandida utilizou o fator de abrangência, $\mathrm{k}=2$. 
Após determinação do valor certificado de propriedade do material INCQS-MRC01, foi elaborado um relatório detalhado de todo procedimento para produção do mesmo, bem como, o certificado do material baseado na norma da Associação Brasileira de Normas Técnicas (2004) ISO Guia 31. Este relatório foi enviado somente aos laboratórios participantes (CARDOSO, 2008).

O conjunto de material de referência produzido é indicado ao uso para os seguintes propósitos: validação e verificação de métodos para determinação de $\gamma-\mathrm{HCH}$, fenitrotiona, clorpirifós e procimidona em hortifrutigranjeiros da classe dos vegetais frutíferos, preparação de material de referência secundário de composição similar e para calibração de equipamentos, na determinação dos agrotóxicos estudados.

\section{Conclusões}

O parâmetro homogeneidade é de grande relevância na produção de um material de referência, por prover informações relativas à qualidade dos resultados analíticos e, conseqüentemente, não devem ser negligenciados. Em virtude disso, as etapas de produção de um material de referência devem ser minuciosamente planejadas, a fim de se garantir o bom estado de conservação do material.

A etapa prévia realizada forneceu dados fundamentais e imprescindíveis à elaboração do material INCQS-MRC01, no que se refere ao tamanho da partícula da amostra, à solubilidade do solvente indicado para preparo da solução de fortificação e ao uso de soluções individuais dos agrotóxicos, que garantiram a homogeneidade do lote produzido.

A partir das condições ideais definidas para o preparo do material de referência preparou-se o material INCQS-MRC01. A incerteza associada à homogeneidade do material informa sobre a variabilidade do lote preparado e foi incorporada à incerteza combinada do material INCQS-MRC01. Para a atribuição do valor de propriedades do mesmo seguiu-se a indicação pela elaboração de um estudo interlaboratorial. Apesar da participação de poucos laboratórios nesta etapa foi possível chegar a um valor estatisticamente comprovado.

O objetivo principal deste trabalho foi alcançado com a preparação e certificação do material de referência INCQSMRC01.

\section{Agradecimentos}

À Financiadora de Estudos e Projetos - FINEP pelos auxílios financeiros concedidos ao projeto "Estruturação de uma rede de laboratórios de análise de resíduos de agrotóxicos para apoio à exportação de alimentos (REAGROEX)".

\section{Referências bibliográficas}

ALVES, N. P.; MORAES, D. N. Metrologia química e a utilização de materiais de referência em medições químicas. 2002. Disponível em: <http://www.quimlab.com.br>. Acesso em: 02 fev. 2004.
ARMISHAW, P.; MILLAR, R. A natural matrix (pureed tomato) candidate reference material containing residue concentrations of pesticide chemicals. Fresenius Journal Analytical Chemistry, v. 370, p. 291-296, 2001.

ASSOCIAÇÃO BRASILEIRA DE NORMAS TÉCNICAS. Materiais de referências: conteúdo de certificados e rótulos. Rio de Janeiro: ABNT, 2004. Guia 31.

ASSOCIAÇÃO BRASILEIRA DE NORMAS TÉCNICAS. Requisitos técnicos para a competência de laboratório de ensaios e calibração: ISO/IEC 17025. Rio de Janeiro: ABNT, 2005.

BASTOS, L. H. P. et al. Ensaio de proficiência para análise de ditiocarbamatos em polpa de banana. Quimica Nova, v. 30, n. 1, p. 32-35, 2007.

BRASIL. Ministério da Saúde. Agência Nacional de Vigilância Sanitária. Agrótoxicos e toxicologia. Disponível em: $<$ http://www.anvisa.gov. br/toxicologia.residuos/index.htm>. Acesso em: 06 mar. 2008.

BRASIL. Ministério da Saúde. Agência Nacional de Vigilancia Sanitária - ANVISA. Programa de Análise de Resíduos de Agrotóxicos em Alimentos (PARA). Brasília, DF, 2008. 21 p. Relatório de atividades 2001-2007.

BRASIL. Ministério da Saúde. Agência Nacional de Vigilancia Sanitária - ANVISA. Resolução - RDC n 21, de 26 de janeiro de 2001. Diário Oficial da União, Brasília, DF, 29 jan. 2001. Disponível em: <http://www.anvisa.gov.br/legis/resol/21_01rdc.htm>. Acesso em: 23 fev. 2008.

CARDOSO, M. H. W. M. et al. Aplicação da radiação gama na preservação de material de referência a ser usado na análise de resíduos de agrotóxicos. Analytica, v. 36, p. 64-69, 2008.

CARDOSO, M. H. W. M. Preparação de um material de referência certificado para controle de agrotóxicos em hortifrutigranjeiros. 2008. 191 p. Tese (Doutorado) - Instituto Nacional de Controle de Qualidade em Saúde, Rio de Janeiro, 2008.

CHUI, Q. S. H.; IAMASHITA, C. O.; BISPO, J. M. A. Estudo de homogeneidade de lote de material silício metálico candidato a material de referência. Química Nova, v. 28, n. 3, p. 497-501, 2005.

ELLISON, S. L. R. et al. Uncertainty for reference materials certified by interlaboratory study: recommendations of an international study group. Accreditation and Quality Assurance, v. 6, p. 274-277, 2001.

INTERNATIONAL LABORATORY ACCREDITATION COOPERATION. Guidelines for the requirements for the competence of reference material producers. Silverwater: ILAC, 2000. G12. Disponível em: <http://www.ilac.org>. Acesso em: 11 fev. 2008.

INTERNATIONAL ORGANIZATION FOR STANDARDIZATION. Reference materials: general and statistical principles for certification. Switzerland: ISO, 2006. Guide 35.

van der VEEN, A. M. H.; LISINGER, T.; PAUWLES, J., Uncertainty calculations in the certification of reference materials: homogeneity study. Accreditation and Quality Assurance, v. 6, p. 26-30, 2001.

VITAL, H. C. et al. Experimentos dosimétricos no irradiador gama do IPE. In: ENCONTRO NACIONAL DE APLICAÇÕES NUCLEARES, 5., 2000, Rio de Janeiro. Anais ... Rio de Janeiro: ENAN, 2000.

ZUCCHINI, R. R. Bate-papo programado: materiais de referência certificados. 2002. Disponível em: <http://www.ipt.br.>. Acesso em: 02 fev. 2004. 